\title{
DESARROLLO TERRITORIAL Y GOBERNANZA: REFINANDO SIGNIFICADOS DESDE EL DEBATE TEÓRICO PENSANDO EN LA PRÁCTICA. UN INTENTO DE APROXIMACIÓN FRONÉTICA
}

Joaquin Farinós Dasi ${ }^{1}$

\begin{abstract}
RESUMEN
El presente texto trata de ofrecer una actualizada síntesis desde el punto de vista del autor sobre cómo poder entender y actuar sobre las relaciones existentes entre gobernanza y acción política para un nuevo desarrollo territorial. Partiendo de una amplia aproximación teórico conceptual, a continuación el texto se centra en el análisis de las opciones, condiciones y limitantes de su puesta en práctica. Como hipótesis en el texto se defiende la necesidad y conveniencia, como opción fronética, de ampliar las relaciones gobernanza-economía, predominantes, con una nueva y más potencial, entre política-territorio, especialmente en la escala local y prestando una especial atención a las nuevas relaciones urbano-rurales. Objetiva la mejor gobernabilidad, un desarrollo territorial más justo y en dignidad, mejor democracia y el bienestar/buen vivir de la población.
\end{abstract}

Palabras clave: Gobernanza. Cooperación territorial. Relaciones urbano-rurales. Desarrollo territorial. Planificación estratégica integral. Multiescalaridad.

\section{TERRITORIAL DEVELOPMENT AND GOVERNANCE; REFINING MEANINGS FROM THEORETICAL DEBATE LOOKING FOR BEST PRACTICES. AN ATTEMPT OF PHRONETIC APPROACH.}

\begin{abstract}
Paper updates, from author's point of view, governance understanding and the way to promote a new spatial development model through new styles of governance from political sphere. By departing from a broad theoretical approach, paper deeps in analysis of alternatives, conditions and barriers to put it in practice. As hypothesis, paper defends necessity and convenience, as fronetic option, to enlarge predominant focus on governanceeconomy relationships with a new more potential one based on political-territorial instead, by focusing at local scale and paying special attention to new urban-rural partnerships; in order to achieve appropriate governability, balanced spatial development in dignity, democracy and citizens' welfare/'buen vivir'.
\end{abstract}

Key words: Governance. Territorial cooperation. Urban-rural partnerships. Spatial development. Strategic spatial planning. Multi-scalarity. ${ }^{1}$ Catedrático de Análisis Geográfico Regional de la Universitat de València. Espanha. E-mail:
Joaquin.Farinos@uv.es 


\section{GOBERNANZA: CONCEPTO Y ¿SÓLO RETÓRICA?}

Ya desde hace más de una década (FARINÓS 2004, 2005, 2008a, 2009a, 2010, 2011c, 2014a, 2015a) venimos trabajando sobre el concepto de gobernanza en relación con el desarrollo territorial y su planificación. Sobre la amplia idea de la gobernanza territorial y urbana como medio, más que como fin en sí mismo; más por tanto como proceso que como precondición, estructura o resultado, para una adecuada planificación, gestión y evaluación de las políticas territoriales o con impacto territorial. Agradeciendo la amable invitación del coordinador de la presente publicación, y en el marco de la 'Rede Iberoamericana de Estudos sobre Desenvolvimento Territorial e Governança', el presente texto trata de ofrecer al lector una actualizada síntesis desde el punto de vista del autor sobre cómo poder entender y actuar sobre las relaciones existentes entre gobernanza y acción política para un desarrollo territorial mejor, más justo, postmoderno, que recupere y valorice localidad y ciudadanía en un mundo, no hay duda, global... pero necesariamente 'glocal'.

Si es que lo que importa de verdad son las personas, la gente, los ciudadanos, y su espacio de vida; ahora necesariamente multiescalar, multiterritorial... de pertenencias múltiples, de espacios de geometrías flexibles y pertenencias múltiples que facilitan las Nuevas Tecnologías de la Información y la Comunicación (NTICs), las infraestructuras y los medios de transporte, y el comercio y los flujos de capital globales. Y es que los gobiernos locales participan de una amplia red de interrelaciones que les resultan imprescindibles. Si las comunidades locales, con capacidades y competencias limitadas y compartidas con otros niveles de gobierno, quieren ser responsables de su propio destino mediante su propia repolitización, necesitan necesariamente de la colaboración de otros poderes. Es la escala local la más compleja de planificar y a la hora de tomar decisiones, porque a ella le afecta todo lo que sucede en el resto de niveles, como ya se planteaba en la década de los años 1980 por parte de la Escuela de Los Ángeles y desde los 'Estudios de localidades'. De ello se desprende, a pesar de la renovada e indiscutible importancia de lo local, que en las actuales condiciones, en pleno siglo XXI y el territorio-red, las polis autárquicas y los neofeudalismos no van a poder regresar, pero lo local sigue siendo protagónico.

El Diccionario de la Real Academia de la Lengua Española incluyó una nueva definición de gobernanza en su $21^{\mathrm{a}}$ edición: "arte o manera de gobernar que se propone como objetivo el logro de un desarrollo económico, social e institucional duradero, promoviendo un sano equilibrio entre el Estado, la sociedad civil y el mercado de la economía"; a diferencia de "gobernabilidad", como originalmente y de forma errónea se tradujo del inglés al castellano el anglicismo "governance". Gobernabilidad se refiere a las "las condiciones políticas para intermediar intereses y el apoyo político para gobernar", de conseguir un estado de derecho en que todos se encuentran sometidos a la ley por igual, y se acepta, aunque haya grupos o individuos que tuvieran posibilidad de evitarlo. Podría decirse que la gobernanza es una condición necesaria, pero no suficiente, para la gobernabilidad, el fin último para una vida comunitaria en armónica convivencia; y ambas difieren a la vez del 'gobierno', acción que desempeña la gobernación (los representantes del poder político) (FARINÓS, 2015a; ROMERO; FARINÓS, 2011) .

Como ya decíamos (FARINÓS, 2008a) el concepto de gobernanza tuvo su origen en la economía institucional y de la regulación. Como hemos señalado (FARINÓS; FERRÃO, 
2015) se empezó a utilizar en los EE.UU. tras la $1^{\text {a }}$ Guerra Mundial para referirse a las nuevas formas de regulación de las actividades económicas basadas en decisiones concertadas entre empresas. El término se generalizaba a partir de la década de los años 1980 y consolidaba a principios de los años 90, cuando irrumpió ampliamente en el discurso político ${ }^{2}$. Como hemos escrito (FARINÓS, 2008a/b) ha sido vista con recelo por su sospechosa procedencia de cara a mantener la defensa del interés general (y no sólo el de los grupos hegemónicos) y dudosa reputación (por presuponer una progresiva tendencia a la desregulación y relajación del papel del estado frente al mercado) $)^{3}$.

Nacía, así pues, con el objetivo de simplificar los procesos de regulación y de intervención de los poderes públicos, y de facilitar la toma de decisiones del resto de agentes sociales, sobre todo los económicos. Desde el mundo de la empresa, la gobernanza se encuentra estrechamente ligada a la toma de decisiones. Se trata de gestionar y dirigir los negocios de forma que se obtenga la máxima eficacia económica. Algunos de los trabajos e indicadores de gobernanza, por ejemplo los desarrollados por el Banco Mundial, serían un claro ejemplo de este enfoque.

Primero referido al ámbito empresarial (gobernanza corporativa) y a la administración pública (RHODES, 1999; STOKER, 1998), más tarde llegaría a las relaciones internacionales (gobernanza internacional, multinivel, buena gobernanza), a las políticas públicas (gobernanza en red, para la sostenibilidad, gobernanza territorial) y al funcionamiento de la vida colectiva (gobernanza democrática, gobernanza social), en el marco del pretendido tránsito desde el paradigma del gobierno al de nueva gobernanza. Ha cobrado a partir de entonces un sentido claramente politológico (formas de ejercer la labor de gobierno y de toma de decisión - PRATS, 2001, 2005; SUBIRATS, 2015-) y administrativista (estructura de los estados y coordinación entre los distintos niveles de gobierno - gobernanza multinivel- vid. HOOGHE; MARKS, 2001; MARKS; HOOGHE, 2004).

La atención se fija a partir de entonces en los cambios clave en los gobiernos actuales y en el replanteamiento del Estado y la pluralidad de actores en la toma de las decisiones públicas; el tránsito hacia el nuevo gobierno-red que apuntaba Castells (RHODES, 1999, 2000). La dirección jerárquica da paso a nuevas formas de regulación basadas en la negociación y la coordinación, mediante las cuales los responsables públicos tratan de movilizar junto a los actores públicos y privados los recursos y potenciales existentes en el territorio.

El papel del estado en la nueva sociedad-red deviene más complejo y difícil. Más allá del gobierno tradicional, suministrador o facilitador, este se va convirtiendo en mediador y

\footnotetext{
${ }^{2}$ En 1985 J.R. Hollingsworth y L.N. Lindberg, dos autores considerados un referente, publicaban «The Governance of the American Economy: The Role of Markets, Clans, Hierarchies, and Associative Behaviour». Ya en la década de los 90 publicaban otra de sus referencias básicas (LINDBERG, CAMPBELL y HOLLINGSWORTH, 1991).

${ }^{3}$ Con afiladas críticas de 'think-tank' próximos a opciones de izquierda como Le Monde Diplomatic; también de los defensores de un modelo de estado de bienestar o modelo tradicional europeo frente al proyecto de mercado libre anglosajón, y al progresivo debilitamiento de Estado a partir de las reformas del sector público (la mal llamada 'Nueva Gerencia Pública') que desde planteamientos neoliberales se planteaban y pusieron en marcha en los años ochenta (con claros exponentes como Ronald Reagan y Margaret Thatcher). Unas propuestas que harán su reaparición, revisitados, en la primera década del siglo XXI, coincidiendo con la gran crisis económica y financiera iniciada en 2007 por la que seguimos transitando. Volvemos sobre esta cuestión, de la utilización de la gobernanza para reinterpretar las relaciones entre estado y mercado, en el segundo punto del texto.
}

DRd - Desenvolvimento Regional em debate (ISSNe 2237-9029) 
líder de procesos, en gestor de conflictos y productor de acuerdos entre los diversos intereses existentes pero bajo su propio horizonte, meta o destino que tiene previsto. Esto en el mejor de los casos, cuando no queda reducido a un mero gestor de la diferencia en un 'game management' o como correa de transmisión de los intereses hegemónicos en el marco de una mera economía política. Ello permite transitar como un continuo, sin romper el nexo entre ellos, del gobierno a gobernanza (FARINÓS, 2008a; PIERRE, 2000), y de la democracia meramente formal (secuestrada - FARINÓS, 2015b) a una democracia participada (real), a una nueva gobernanza democrática.

La noción de gobernanza posibilita una nueva perspectiva para analizar la complejidad del proceso de toma de decisiones generado por la pluralidad de actores (Estado, del mercado y la sociedad civil) que participan, con intereses distintos y hasta contrapuestos, en el proceso de toma de decisiones (MAYNTZ, 2001; MORATA; HANF, 2000, PETERS, 2010). De acuerdo con Prats (2001) la gobernanza es multifacética y plural, busca la eficiencia adaptativa y exige flexibilidad, experimentación y aprendizaje por prueba y error; o como también señalan Arrellano, Sánchez y Retana (2014, p. 11) "[...] la gobernanza como instrumento será susceptible a fallas de implementación y sus resultados dependerán, en gran medida, de la forma concreta en que se diseñe, instaure y lleve a cabo". Desde este punto de vista, la gobernanza es un proceso más indicativo que genérico, basado por tanto en las propias especificidades del contexto ('path dependency' geográfica e histórica) que se adaptará a cada territorio en función de sus características, y en el que la colaboración y la flexibilidad resultarán aspectos clave.

El obligado ajuste para poder acoplar mejor la organización territorial del estado a las nuevas necesidades funcionales en el actual contexto global obliga a transitar del gobierno (como estructura o precondición -más rígida y menos flexible-) a la gobernanza (como proceso) a la hora de elaborar e implementar las políticas públicas con impacto sobre el territorio. De este modo, en el actual contexto se ha producido un cambio en la forma de hacer política. Las políticas no se imponen a los territorios (unos territorios que se adaptan a las exigencias de las mismas, y a las del capital y la inversión extranjera directa) sino que son las políticas las que se adaptan a los territorios, con un claro enfoque 'local based' (BARCA, 2009).

Son los territorios los que deciden y hacen política, a partir de un claro proceso de repolitización, que cabe relacionar tanto con procesos de descentralización como de participación y empoderamiento. Es lo que algunos autores han dado en llamar 'inteligencia gubernamental', instrumento para la gestión de la información y el conocimiento necesario para una adecuada gobernanza del territorio. Las políticas públicas se adaptan al territorio y no a la inversa; ello obliga a una consideración multiescalar (multinivel) en la toma de decisiones para dotar de coherencia, encontrando sinergias y complementariedades, entre las actuaciones impulsadas desde los distintos niveles. 
Figura 1 - Las cuatro dimensiones de la Gobernanza Territorial (hacia la Gobernabilidad)

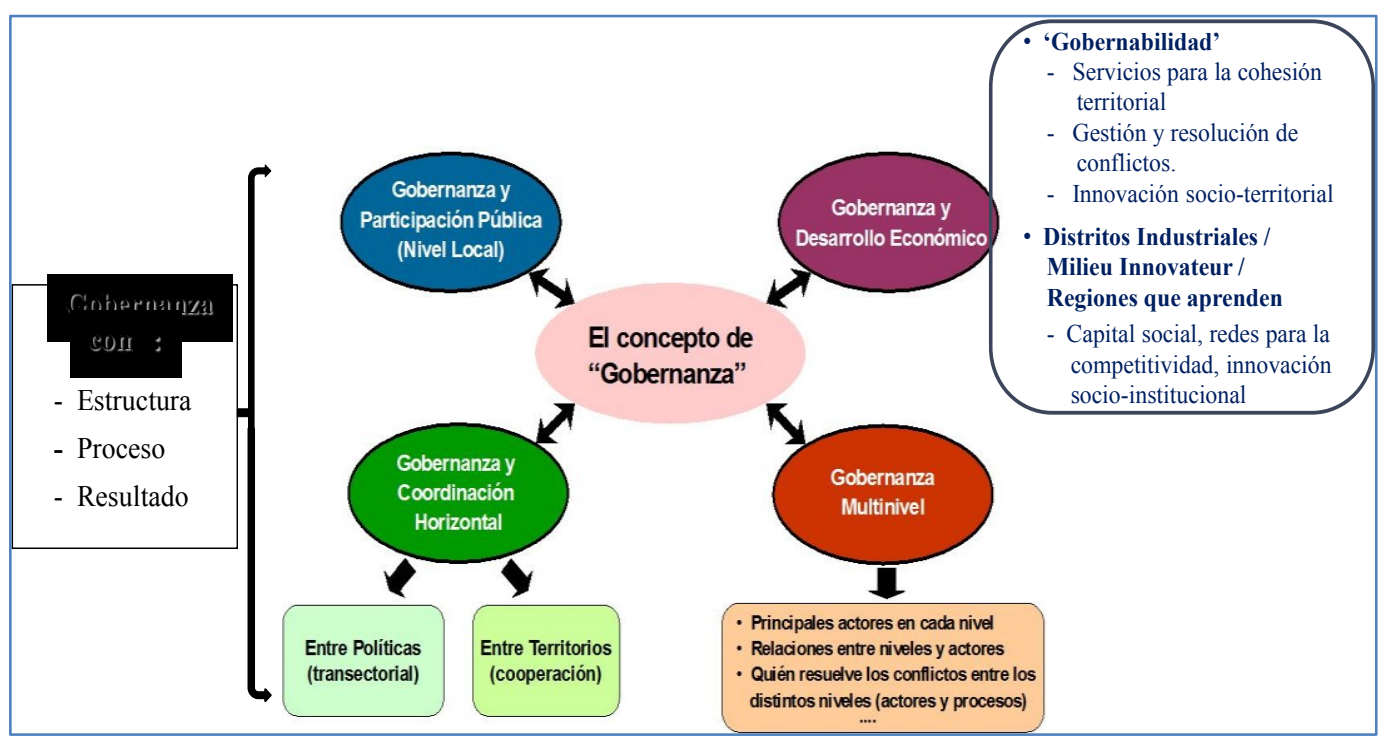

Fuente: El autor

Todo ello nos aboca a nuevas formas de gobernanza (FARINÓS, 2005a y 2008, FARINÓS; FERRÃO, 2015), en sus distintas dimensiones (vid. figura 1): vertical/multinivel, respetando los principios de subsidiariedad y proporcionalidad de acuerdo con las competencias y recursos disponibles (la cuestión crucial no es ya tanto quién gobierna, quién tiene los recursos, sino cómo lograr una gobernabilidad eficiente y que las políticas consigan sus objetivos); horizontal (coordinación entre políticas sectoriales y entre territorios cooperación territorial- para llegar a dotarlas de la coherencia necesaria); de participación/democracia deliberativa eficiente que conduzca a una gobernabilidad real, a un verdadero estado de derecho, donde la transparencia y la rendición/dación de cuentas a los usuarios (mediantes los pertinentes sistemas y prácticas de evaluación y seguimiento de las actuaciones - FARINÓS, 2011a/b), la llamada 'inteligencia territorial', pasen a ser principios elementales e ineludibles. Sobre ello volveremos más en detalle en el tercer y último apartado de este texto.

\section{ESTADO - MERCADO (ECONOMÍA Y POLÍTICA), LA RELACIÓN PRIMADA DE LA GOBERNANZA; RIESGOS E INCONGRUENCIAS DE UN PLANEAMIENTO INCOMPLETO DE INGOBERNABILIDAD}

En la sección anterior se ha visto cómo el concepto de gobernanza ha ido adquiriendo carta de naturaleza y cada vez más trascendencia en los debates conceptuales y teóricos, pero también en la práctica política, abriendo el campo a nuevas formas de llevar a cabo la acción de gobierno y de gestionar las políticas públicas, promoviendo la reforma del sector público. La crisis económica y financiera, la actual y otras previas motivadas entonces y también por el endeudamiento de países en vías de desarrollo, ha contribuido a reconducir la aproximación 
a la idea de gobernanza como un elemento o factor clave para el desarrollo económico ${ }^{4}$. Éste se interpreta, como también y por ende la cooperación al desarrollo, con unos planteamientos tradicionales que lo equipara a modernización, siguiendo de forma mimética (banal y uniforme) patrones occidentales de democracias liberales y de mercado. Cabe plantear alternativas a esta interpretación, parcial y finalista de la gobernanza, que gana en amplitud y plenitud con un modelo de desarrollo basado más en la cultura y la identidad, en lo local y en el propio potencial de las comunidades, 'local based', más participado y democrático (FARINÓS, 2014b, 2014c, 2015b) y con un enfoque más centrado en las relaciones entre geografía/territorio y política en su lugar (FARINÓS, 2014a). Sobre esto último volveremos en el epígrafe siguiente, en éste trataremos de explicar las limitaciones que presenta un enfoque de la gobernanza que hemos llamado 'económico-político', hegemónico en el actual contexto, pero entendemos que insuficiente e ineficaz para un adecuado desarrollo y gobernabilidad democrática.

Según Zurbriggen (2011) existen diferencias entre el enfoque y el desarrollo dado a la gobernanza en América Latina y el que se ha dado en Europa. Desde nuestro punto de vista, y en relación con este enfoque de gobernanza como relaciones entre economía y política, se ha dado una progresiva tendencia a la homogeneización. Cabe entender, sin embargo, que existen en el ámbito de América Central y del Sur algunas particularidades que cabe reseñar.

En América Latina, el debate académico sobre la gobernanza ha respondido al criterio de las instituciones protagonistas de la cooperación internacional. Nos referimos al Banco Mundial, al Programa de las Naciones Unidas para el Desarrollo (PNUD) y al Banco Interamericano de Desarrollo (BID). Estas instituciones, en conjunto, han insistido en la noción de 'buen gobierno'; en un intento de dación de seguridades (en especial para el capital de inversión y 'de riesgo') y en la lucha contra la corrupción. Lo que se pretende es lograr una mayor efectividad de la ayuda internacional (GRINDLE, 2007; WEISS, 2000). De forma paralela, aunque con algún matiz, a la interpretación del Economic Global Forum y sus sucesivos informes sobre la competitividad global.

El Banco Mundial define gobernanza (identificada como buen gobierno) como el conjunto de procesos e instituciones a través de las cuales se determina la forma en que se ejerce el poder en un país, para desarrollar sus recursos económicos y sociales. El buen gobierno se asocia a la transparencia y a la eficacia en tres ámbitos fundamentales: los métodos de elección, control y recambio de los gobernantes (estabilidad institucional); la capacidad del gobierno para administrar los recursos y aplicar las políticas (marco regulatorio y eficacia del gobierno); y el respeto a los ciudadanos (transparencia, participación y garantía del Estado de derecho) (WORD BANK, 2005, p. 3-7). Por su parte el PNUD (1997) la define como "[...] el ejercicio de la autoridad económica, política y administrativa para administrar los asuntos de un país a todos los niveles de gobierno... comprende los mecanismos, los procesos y las instituciones a través de las cuales los ciudadanos y los grupos articulan sus intereses, ejercen sus derechos legales, cumplen sus obligaciones y resuelven sus diferencias". Una buena gobernanza se caracterizaría por ser participativa, transparente, con control público, efectiva, equitativa, promotora de un verdadero Estado de derecho en el que las

\footnotetext{
${ }^{4}$ Homogenizando también situaciones y efectos del llamado Consenso de Washington, antes aplicado en países latinoamericanos, ahora al territorio europeo a través de la llamada Troika (Banco Central Europeo, Fondo Monetario Internacional y Comisión Europea).
}

DRd - Desenvolvimento Regional em debate (ISSNe 2237-9029)

v. 5, n. 2, p. 4-24, jul./dez. 2015. 
prioridades políticas, sociales y económicas se basan en un amplio consenso de toda la sociedad (una gobernabilidad real, como ya hemos comentado) . $^{5}$

Lo que subyace detrás de todo ello es la defensa de un modelo de Estado, y la necesidad de fortalecerlo para que puedan desenvolverse las fuerzas del mercado de la forma más eficaz y eficiente para un adecuado crecimiento económico (y por ende desarrollo) de los países y sus regiones. Se sigue por tanto con una interpretación clásica del concepto de desarrollo que lo identifica como proceso de modernización. En este proceso se establece el reparto de funciones entre lo que (y no tanto para quién) debe hacer el Estado; cada vez menos según los presupuestos neoliberales, que pretenden transferir sus funciones todo lo posible al mercado ${ }^{6}$. La mayor participación del sector privado y la sociedad civil en actividades que hasta ahora estaban reservadas al sector público se basa tanto en la búsqueda de una mayor eficacia, supuestamente para ajustarlas mejor a las demandas reales de forma más democrática (pero poniendo el foco más en lo individual que colectivo; liberal por tanto). En suma, se trata de exponer a los organismos estatales a una competencia mayor con el mercado, con el fin de incrementar su eficacia y eficiencia, sustituyendo el modelo jerárquicoburocrático por una nueva gerencia pública; poco neoinstitucional y también claramente alejada de la opción del universalismo comunitario y la autogestión.

Volviendo a la afirmación de las últimas líneas del párrafo previo al inmediato anterior, esto equivale según Prats (2001, p. 114), a interpretar la gobernanza como “[...] el marco de reglas, instituciones y prácticas establecidas que sientan los límites y los incentivos para el comportamiento de los individuos, las organizaciones y las empresas". Dos observaciones a este su comentario. La primera: no todas las esferas de la vida del individuo pueden ser reguladas, ni dan resultado aunque lo sean (como el mismo Prats reconoce). Diálogo, pacto, concertación, acuerdo... abren necesariamente paso para poder lograr la gobernabilidad. La segunda: este modelo, supuestamente 'útil' para los países en desarrollo que reciben ayuda internacional para facilitarlo, ha sido devuelto, como boomerang, a los países occidentales afectados por la actual crisis financiera, en un alarde y demostración de fuerza del pensamiento único. La cuestión es si es posible una alternativa posible y eficaz.

La situación actual que viven muchos de los países occidentales considerados tradicionalmente 'desarrollados', derivada de la actual crisis financiera a escala global, y sus graves impactos sobre sus poblaciones, ha dado lugar a una renovada interpretación del concepto de resiliencia (evolucionista y no). El concepto, como en muchos otros casos, pasa desde las ciencias naturales, desde lo ecológico, donde el concepto tiene su origen, a las

\footnotetext{
${ }^{5}$ Para un mayor detalle en el análisis de la interpretación de la gobernanza desde estas posiciones y para este contexto vid. Tutistar y Pinazo-Dallenbach (2015).

${ }^{6}$ En este sentido cabe citar las iniciativa de la llamada 'Gobernanza Local' (ZURBRIGGEN, 2011, p. 49), una decidida apuesta por procesos de descentralización y transferencia de funciones y competencias (no siempre más bien casi nunca- acompañadas de los recursos necesarios) hacia niveles subnacionales. Esta descentralización, se supone, sería la forma de hacer los servicios públicos locales más eficaz, eficiente y ajustada a las demandas reales de las comunidades, incentivando así su seguimiento y la rendición de cuentas ante los ciudadanos y votantes. Ello se traduciría, también, se presuponía, en la extensión y el fortalecimiento de las instituciones democráticas, y en mayores incentivos para el desarrollo económico local. La gobernanza local aparecía como un instrumento relevante de gestión para alcanzar esos objetivos. La falta de fondos con que hacer efectiva la prestación de los servicios, y la consiguiente dependencia de la iniciativa privada, que tiene sus propias prioridades, como también de los adecuados equipos técnicos en los niveles subestatales de la administración, han resultado ser barreras infranqueables hasta la fecha, por lo que los gobiernos locales siguen manteniendo la dependencia respecto del gobierno central.
}

DRd - Desenvolvimento Regional em debate (ISSNe 2237-9029) 
ciencias sociales. A lo económico primero (recuperación de la crisis, adaptabilidad, innovación tecnológica y su relación con la innovación socio-institucional...), a lo social después, finalmente a lo territorial (innovación socio-territorial, con todas las limitaciones y resistencias a las que ésta se enfrenta al presuponer cambios en las relaciones de fuerza y de poder - ALBRECHTS, 2009).

Es así que se empieza a hablar, también, de 'resiliencia institucional'; lo que a su vez nos devuelve al análisis de las instituciones (y de las organizaciones, del gobierno y del gobierno-red y la gobernanza) y a la idea de 'vulnerabilidad institucional'. Dicho de otro modo, la resiliencia institucional es un nuevo enfoque adicional desde el que poder abordar las relaciones de meta-gobernanza y el papel que juegan las instituciones, tanto a la hora de definir el marco de estas relaciones como de desarrollar las nuevas prácticas de gobernanza (SAPOUNTZAKI, 2015).

De especial interés le resultan las relaciones que se establecen entre los distintos actores territoriales y entre ellos y las instituciones (la gobernación), a menudo traducidas en acuerdos y coaliciones. En lo que se refiere al proceso de planificación para el desarrollo de las políticas acordadas o decididas, las nuevas rutinas de acuerdo y gobernanza, caracterizadas por su flexibilidad, fluidez, adaptabilidad, contingencia, conectividad, multiplicidad... se consideran por algunos autores como testimonio de esta resiliencia (DAVOUDI; STRANGE, 2009). Como también lo es la capacidad (fenomenológica) de poder aprender de la experiencia, de los éxitos (buenas prácticas que lleguen a permitir incluso el establecimiento del 'benchmarking') y de los fracasos, a la hora de adaptar las instituciones y los procedimientos de planificación y prepararlos para futuras perturbaciones (inevitables) (LU; STEAD, 2013) ${ }^{7}$.

Así pues, la buena gobernanza se ha venido considerando por la literatura politológica, geográfica y económica como un elemento clave del desarrollo y, casi de forma metonímica respecto de él, para la competitividad territorial y urbana. Nuestro punto de vista es crítico, y alternativo, respecto de este credo, entendiendo la gobernanza como un importante factor para hacer posible un renovado entendimiento del desarrollo (basado en las especificidades locales), donde importa menos la competitividad excluyente (de acuerdo con la teoría de juegos de suma cero) que la competitividad colaborativa ('win-win') y el principio de la equidad (equilibrio) territorial. Asumiendo que el espacio 'per se' es anisotrópico (aquello de la síntesis regional única de la geografía regional francesa clásica, la cultural de Sauer desde el ámbito anglosajón y la social-relacional de la visión postmodernista). La igualdad por lo tanto no ha lugar... a no ser que se pretenda una estandarización y homogeneización que facilite la banalización y la incapacidad de los territorios para poder desenvolverse mediante una adecuada gestión y negociación en el uso de sus recursos no genéricos sino específicos... a partir los que poder desarrollarse; bien sea a partir de los factores propulsivos básicos, los sectores motor y los efectos de retroalimentación y de generación de una red de actividades que convengan, bien a partir de procesos de innovación social y tecnológica.

A través de la cooperación territorial pueden encontrarse las economías de escala (también el poder de presión e influencia) y la eficiencia necesarias que superen muchas de

\footnotetext{
${ }^{7}$ Ciudades y territorios resilientes son aquellos que desarrollan capacidades que les ayudan a asimilar futuros impactos y presiones sobre sus sistemas sociales, económicos, tecnológicos, y sobre su infraestructura, hasta el punto de hacerles capaces de mantener esencialmente sus mismas funciones, estructuras, sistemas e identidad (tomado y adaptado de http://www.resilientcity.org/index.cfm?pagepath=Resilience\&id=11449).
}

DRd - Desenvolvimento Regional em debate (ISSNe 2237-9029) 
las limitaciones del minifundismo (Farinós 2009b, 2013). Esta cooperación requiere de las distintas dimensiones de la gobernanza, en especial multinivel, de cooperación entre territorios, limítrofes y no, tanto para la prestación de los servicios de interés general para los ciudadanos como para el diseño y puesta en práctica de las políticas. Idealmente a partir de una nueva planificación territorial estratégica comprehensiva o integral (FARINÓS, 2010; PASCUAL, 2011).

Se trata de pasar, como se ha dicho, de la territorialización de las políticas a las políticas de desarrollo territorial desde los territorios. Hay más que indicios de que más de lo mismo no será suficiente (FARINÓS, 2011c) y que un nuevo modelo de desarrollo alternativo basado en las localidades y el valor del territorio (un verdadero sistema complejo integrado por soporte, objetos, población, recursos, potenciales y sus relaciones - SANTOS, 1996 -, diversas y hasta contradictorias, como es natural en sistemas complejos) no sólo es posible sino también recomendable (FARINÓS, 2014b) y hasta de sentido común (fronético - vid. FARINÓS; VERA, 2015-). A ello dedicamos el epígrafe que sigue.

\section{RECONSIDERANDO LAS RELACIONES ENTRE TERRITORIO Y POLÍTICA; LA GOBERNANZA TERRITORIAL COMO ALTERNATIVA DE 'GOBERNANZA PLENA' Y COMO GOZNE PARA UN NUEVO DESARROLLO TERRITORIAL EN DIGNIDAD}

La alternativa que planteamos en este epígrafe respecto del anterior se enmarca claramente en el enfoque geográfico, tomando el territorio (y la sociedad que lo produce) como elemento clave a la hora de decidir sobre el futuro deseado y la mejor forma de poder conseguirlo (esto presupone per se una mejor gobernabilidad). El territorio y su planificación (del desarrollo territorial) se convierten en campo predilecto de aplicación o puesta en práctica de los principios de buen gobierno y de gobernanza. Se aboga por tanto por una renovada relación entre politología y geografía, como forma de poder saldar la distancia entre Estado y ciudadano, hacer efectivo el contrato social entre gobernantes y ciudadanos, y mejorar las relaciones de metagobernanza entre Estado, mercado y sociedad civil para poder garantizar, al fin, el Estado de derecho y la gobernabilidad. Nuestra tesis: las nuevas formas de planificar y actuar sobre el territorio, ayudan a lograr esta meta (FARINÓS, 2014b). En esta relación, con un enfoque de repolitización, encuentra su acomodo y se integra, cómo no, la dimensión de la economía, pero esta vez desde unos planteamientos 'territorializados', en línea con lo que se ha dado en llamar la nueva geografía económica 'evolucionista', una denominación que tal vez no sea demasiado acertada y que se debiera aplicar más a la economía que no a la geografía, plasmada en una reconsideración del enfoque a seguir desde la Ciencia Regional, tal y como ya apuntaba George Benko (1998) y se plantea, por parte de algunos sectores de economistas (vid. GALLEGO; PITXER, 2015). Un nuevo campo de interés y aplicación para la geografía (más política, regional y territorial - FARINÓS, 2015c, 2014d-), para el estudio de las relaciones multiescalares en nuevas multi-territorialidades, sentidos de pertenencia y espacios de vida (con geometrías variables y fronteras difusas -Farinós 2001- provocando desajustes entre territorialidad y territorio) y para la consideración del territorio con un enfoque de sistema complejo (MOINE, 2006). 
¿Qué aporta esta visión territorial al ámbito de la ciencia política? Martín (2015) nos permite responder esta cuestión a partir de la respuesta que da a otras tres que él mismo se plantea: qué territorios están representados en el Estado (lo que podríamos relacionar con la 'polity', y en su opinión tiene que ver con los procesos de input en un sistema político); qué poder tiene la representación de cada territorio (que podríamos aproximar a la 'politics', y según él se refiere a la "toma de decisiones en el centro neurálgico del sistema"); cómo se articula la política desde la perspectiva territorial (que relacionamos con la 'policy' y él con "los outputs o políticas públicas que tratan de responder a las demandas recibidas"). Es la tercera la que más me ha venido interesando en mi labor de investigación sobre gobernanza, partiendo de la idea de que lo verdaderamente importante desde el punto de vista del servidor público y el planificador son las políticas (las 'policies') y sus resultados o efectos a la hora de satisfacer las necesidades de la población; tanto de la sociedad civil en general como de los grupos de interés específicos. Al servicio de las 'policies' deberían estar tanto 'politics' (mecanismos y estructuras de toma de decisiones) como la 'polity' (organización territorial y modelo de estado, precondición o estructura para el buen gobierno y la gobernanza) ${ }^{8}$.

La oportunidad de este interés por los procesos mediante los que se diseñan e implementan las políticas (y la búsqueda de nuevas prácticas de buen gobierno y gobernanza, esta vez entendida como proceso y ya no como estructura o precondición -vid. la figura 1) queda refrendada al comprobar que "[...] desde la perspectiva normativa del derecho constitucional, sin embargo, sólo las dos primeras han sido atendidas y estudiadas en términos de división territorial del poder o articulación territorial del Estado" (MARTíN, 2015).

La adaptación de las formas de organización territorial clásicas a las nuevas necesidades funcionales surgidas de los procesos de cambio global requiere también, si no antes, adaptar las viejas formas de elaborar e implementar las políticas públicas. Como decíamos párrafos arriba, no se trata de imponer políticas a los territorios sino de hacer política desde los territorios. Ello implica cambios en el proceso de toma de decisiones pero también, y consecuentemente, en la forma en que los temas entran en la agenda política, la forma en que se evalúan los resultados; en suma, en el estilo de hacer política. Ello es posible gracias a la emergencia de lo que se ha dado en llamar, asociada al concepto de gobierno-red, 'inteligencia gubernamental': instrumento de gestión de la información y del conocimiento necesario para llevar a cabo una buena gobernanza del territorio 9 .

Esta inteligencia gubernamental cabe ponerla en relación o, mejor, entenderla como parte integrante de la llamada 'inteligencia territorial', a su vez uno de los máximos exponentes del llamado capital territorial inmaterial, uno de los recursos y potenciales para el desarrollo más sustantivo. Los orígenes del concepto de inteligencia territorial se sitúan a finales de los años 1980, en el marco de proyectos de lucha contra la pobreza. La combinación de tecnocracia (carencia de participación) en la elaboración de los planes, el posicionamiento crítico respecto de los modelos de desarrollo (in)sostenible, en el nuevo

\footnotetext{
${ }^{8}$ También hemos demostrado en el proyecto ESPON 2.3.2 (FARINÓS, 2007) que las estructuras del estado no resultan determinantes o decisivas a la hora de desarrollar nuevas y más eficientes prácticas de gobernanza, más la tradición y la cultura política.

9 Sobre el concepto de inteligencia gubernamental vid. Sánchez de Juan (2004), también los informes y publicaciones del proyecto CaENTI (http://www.territorial-intelligence.eu/portail/site/).
}

DRd - Desenvolvimento Regional em debate (ISSNe 2237-9029)

v. 5, n. 2, p. 4-24, jul./dez. 2015. 
marco que favorecen las NTICs, facilitan la aparición de nuevos métodos basados en dicha inteligencia territorial ${ }^{10}$.

Es el propio Girardot (2010, p. 26) quien nos facilita el entendimiento acerca del concepto de inteligencia territorial, "[...] un medio para los investigadores, para los actores y para la comunidad territorial de adquirir un mejor conocimiento del territorio, pero también de controlar mejor su desarrollo...[...]", al destacar tres aspectos de la misma:

-Su dimensión científica o epistemológica, integrando el conocimiento multidisciplinar y los métodos científicos necesarios para comprender las estructuras, sistemas y dinámicas territoriales (lo que nos devuelve a la idea del territorio como sistema complejo, de la 'transboundary sience', la transdisciplinariedad y la llamada ciencia posnormal (FARINÓS; VERA, 2015).

- La reforzada importancia de los actores del territorio que actúan de forma deliberativa pero ponderada, cabría añadir, de acuerdo a su experiencia y conocimiento disponible, en un proceso de co-construcción y co-participación a través del diálogo y la colaboración.

-No entraremos más a fondo aquí sobre cuestiones relativas al poder, en el sentido que plantea Foucault en su obra (para un mayor detalle vid. FARINÓS; VERA, 2015). Pero sí se quiere señalar la importancia que en este sentido han venido cobrando planteamientos como los de la 'investigación acción participativa' (LEWIN, 1946), las propuestas metodológicas de 'pedagogía popular' de Paulo Freire, cercana a los planteamientos de la 'ecología de saberes' y propuestas como los 'talleres de ciencia', las de 'investigación participativa' de Fals Borda o las de 'planificación estratégica situacional' de Carlos Matus... entre otras muchas alternativas para el desarrollo basadas en el conocimiento y la acción participativa (Martin 2015). Todos ellos resultan enfoques para el desarrollo entroncan con los nuevos planteamientos de la llamada gobernanza democrática (PASCUAL, 2011) ${ }^{11}$.

- La cantidad y calidad de información territorial necesaria disponible, haciendo de la información y el conocimiento la clave del desarrollo. Cobran inusitada importancia las fuentes, técnicas y medios (NTICs entre ellos) a la hora de facilitar el trabajo colaborativo de los actores, permitiendo cubrir mejor la secuencia informaciónconocimiento-opinión-posicionamiento-acción ("Datos-Información-Acción" según

\footnotetext{
${ }^{10}$ A finales de esa década (1989) en Francia surgirá el método Catalyse, que posteriormente, a partir de 1994, se extiende a otros países de la UE y posteriormente (2007) a Latinoamérica (GIRARDOT, 2010).

${ }^{11}$ El objetivo general de la gobernanza democrática es el desarrollo humano del grupo de referencia, bien sea éste de escala local, regional, estatal o supraestatal, para lo cual sólo existe una vía: mejorar la capacidad de acción del grupo en términos organizativos a partir del trabajo en red de todos sus miembros, cuya articulación correspondería a los gobiernos democráticos representativos de cada nivel. De este modo, el prototipo de gestión pública propia de la gobernanza democrática sería la 'gestión relacional': conjunto de métodos y técnicas orientados a gestionar proyectos en red mediante la cooperación pública y privada e interinstitucional. Esto nos devuelve a la nueva planificación territorial estratégica integral y alguna de sus recientes manifestaciones ('New Strategic Spatial Planning', 'Comprehensive Planning', 'Smarth Growth'...).
}

DRd - Desenvolvimento Regional em debate (ISSNe 2237-9029)

v. 5, n. 2, p. 4-24, jul./dez. 2015. 
BOZZANO, 2013, p. 5). Especial atención merece en este sentido la figura de los observatorios territoriales ${ }^{12}$.

Así pues, el desarrollo territorial precisa de alianzas efectivas de todos los actores. No basta con los medios técnicos y herramientas ya disponibles en organizaciones e instituciones. Se requiere de un nuevo marco teórico, de nuevas herramientas de análisis y prospección territorial, y de nuevos métodos de planificación y de decisión participativas (ALMASA MAZA, 2010). Pero, conviene recordar, no existe una única estrategia universal que pueda ser aplicada a todo espacio o región, independientemente del contexto.

Pike, Rodríguez-Pose y Tomaney (2006, p. 344), yendo de lo general a lo específico, concretan las, en su opinión, cinco tendencias que en materia de desarrollo local y regional se están produciendo en el actual contexto global: innovación, conocimiento y aprendizaje; crecimiento endógeno; desarrollo sostenible; desarrollo autóctono; post-desarrollismo. En conjunto contribuyen a moldear en cada caso un determinado modelo de desarrollo local (pero dentro de un contexto global) concebido como un "[...] proceso en el que una sociedad local, manteniendo su propia identidad y su territorio, genera y fortalece sus dinámicas económicas, sociales y culturales, facilitando la articulación de cada uno de estos subsistemas, logrando mayor intervención y control entre ellos". No todas las iniciativas resultan fáciles de implementar ni, mucho menos, plenamente exitosas, al tener que enfrentarse a múltiples problemas y limitaciones. Sin embargo, mediante su repolitización y con un carácter más proactivo, las comunidades locales (y sus redes de cooperación a distintos niveles, en distintos ámbitos y con distintos contenidos) pueden tratar de influir y decidir sobre su propio futuro (liderazgo frente a fatalismo).

El Estado-Nación ha dejado de ser el garante del desarrollo y de la competitividad de las economías nacionales o, al menos, lo ha dejado de ser en exclusiva. Necesita del mercado y de la sociedad civil, desde los planteamientos de la nueva gobernanza, y del resto de niveles de gobierno, infra y supra estatales, desde el punto de vista de la administración, gobierno o gobernación ${ }^{13}$. Por ello, tal y como hemos venido insistiendo, deben reorganizarse sus relaciones: vertical y horizontalmente.

En los párrafos y secciones precedentes nos hemos referido y prestado mayor atención a algunas de las dimensiones de la gobernanza tales como la coherencia intersectorial de las actuaciones (planificación territorial integral), al papel de la participación y del empoderamiento para una gobernabilidad real que otorgue seguridades para un nuevo modelo de desarrollo sostenible y en dignidad de cada territorio dentro del actual sistema global; también a la coordinación multinivel. Ahora, para cerrar el texto, nos queremos centrar en un aspecto concreto de la dimensión horizontal de la gobernanza: la cooperación territorial y, dentro de ella, a las relaciones urbano-rurales. A nuestro juicio resulta un elemento indiscutiblemente clave para el objetivo del desarrollo territorial equilibrado, pero parece estar siendo soslayado por el predominio o imposición, de forma excluyente, del valor de la ciudad

\footnotetext{
12 Sobre los observatorios territoriales su papel, funciones, requisitos, oportunidad y funcionamiento pueden verse, entre otros, Farinós, 2011a,b y 2009c, Valenzuela, 2015 y 2011.

${ }^{13}$ Un gobierno que pasa de ser "unidireccional", estado-céntrico y jerárquico (gobernantes-gobernados), a "bidireccional" (público-privado) (KOOIMAN, 2003), y más heterárquico (sin soslayar el poder y los grupos que lo ostentan e incluso detentan); donde la gobernanza se entiende como un fenómeno social y de colaboración para presentar soluciones y oportunidades colectivas en el marco de una gobernabilidad real y efectiva.
}

DRd - Desenvolvimento Regional em debate (ISSNe 2237-9029)

v. 5, n. 2, p. 4-24, jul./dez. 2015. 
sobre el territorio, de la competitividad territorial sobre la cohesión, de lo genérico sobre lo indicativo y particular, de lo hipotético-deductivo sobre lo fenomenológico, del espacio sobre la localidad.

Decíamos que el concepto de equilibrio y cohesión territorial se relaciona con el de policentrismo y el de cooperación territorial. Desde el punto de vista de la prestación de servicios y el desarrollo de las propias competencias sobre el territorio, cada actuación (y servicio a prestar) requiere una escala territorial que le resulta idónea. No es la misma en todos los casos y puede presentar umbrales mínimos, no ya de eficiencia sino de eficacia. Desde este punto de vista la gobernanza es un proceso que se adaptará a cada territorio en función de sus características, y en el que la colaboración y la flexibilidad resultan aspectos clave. Dos son las respuestas que se han querido dar: la aplicación del principio de subsidiariedad y/o el desarrollo de acuerdos de cooperación, interterritorial (inter-municipal) o interescalar (entre varias administraciones y de éstas con la iniciativa privada).

La cuestión de la cooperación intermunicipal guarda relación tanto con la articulación y la cohesión territorial (FARINÓS, 2013 y 2009b) como también con el clásico debate sobre la escala óptima para la prestación de servicios (concentración o descentralización - 'public choice') y los ámbitos territoriales de actuación de los gobiernos. A tenor del análisis de experiencias previas y de las precondiciones para la formulación y posterior aplicación de las estrategias de desarrollo, desde gran parte de la literatura (en materia de planificación) se parte de la idoneidad de la escala supramunicipal y subregional y resulta predilecta. La cooperación intermunicipal, por tanto, se constituye como elemento fundamental para una adecuada gobernanza territorial, por las ventajas de la colaboración en términos de eficiencia y de posibilidad de acceso a los bienes y servicios públicos; pero también por favorecer, de forma coherente con los principios de subsidiariedad y proximidad, el cumplimiento de los principios de apertura, participación, responsabilidad, eficacia y coherencia que caracterizan la llamada 'buena gobernanza' (CE, 2001) ${ }^{14}$.

Más específicamente, desde el punto de vista de la cooperación y, sobre todo, complementariedad urbano-rural, resulta prioritario establecer mecanismos de compensación y estructuras flexibles de gobernanza que posibiliten que los municipios del entorno de un nodo o centro urbano (en especial los de tamaño mediano y pequeño) puedan acceder a la actividad económica y a los servicios capaces de crear unas condiciones mínimas a partir de las que la población pueda promover innovación social e iniciativas autóctonas. A día de hoy, éstas pueden apuntar claramente a nuevas formas de desarrollo alternativas o complementarias del modelo actualmente imperante, e imperfecto, y que precisamente contribuye a perpetuar algunos de sus negativos efectos e impactos sobre comunidades y el planeta.

Volviendo al concepto de policentrismo, éste puede ser interpretado a diferentes escalas, de la mundial a la supranacional y nacional, pero también lógicamente, en las propias regiones, haciendo partícipes a las áreas rurales de un nuevo modelo desarrollo más sostenible $^{15}$. En primera instancia aprovechando adecuada y creativamente el propio

\footnotetext{
${ }^{14}$ A pesar de las dificultades para poder resolver adecuadamente la ecuación a la hora de equilibrar criterios económicos de eficiencia con los políticos de democracia, representatividad y/o empoderamiento.

${ }^{15}$ Parte de la bibliografía reciente de la UE, desde donde más se ha impulsado el concepto de policentrismo, en especial en algunos trabajos de Simin Davoudi, de la Universidad de Newcastle, antes en la de Leeds, el policentrismo a escala europea y estatal (nacional), con la presencia de ciudades de dimensiones eficientes
}

DRd - Desenvolvimento Regional em debate (ISSNe 2237-9029) 
patrimonio natural y cultural (como recurso y potencial territorial) de las mismas; de forma un tanto más evolucionada poniendo en valor los nuevos servicios ecológicos y de infraestructura verde (ampliando el concepto de multifuncionalidad - de agrícola a rural... finalmente a territorial-) que pueden aportarse desde estos espacios rurales.

Así pues, las regiones urbano-rurales que cuentan con un sistema de ciudades de distintos niveles, pero sobre todo con pequeñas y medias, pueden articular una red de territorios que les permitan a la vez: actuar cooperativamente aportando los servicios fundamentales (de interés general) a las zonas rurales próximas de toda la región, al tiempo que los zonas rurales aportan sus propios servicios, recursos y calidades a su región (y a la población urbana). No resulta fácil concretar la forma de poder hacerlo, más allá de la habitual retórica sobre los valores antropológicos y paisajísticos de estos espacios, pero cada vez es posible concretar más. Por ejemplo se va imponiendo progresivamente el criterio de los mercados de proximidad para el producto diferenciado y sostenible (ecológico incluso) capaz de mantener en el campo la población dedicada a actividades, bienes y servicios vinculados, en primera instancia, a las ciudades próximas. Bajo este supuesto sería posible configurar un nuevo espacio de desarrollo más imbricado, cohesionado y diverso (vid. el tercer escenario, 'C', del proyecto ESPON Scenarios 2050 en comparación con el 'B', policéntrico, y sobre todo al 'A', metropolitano - http://www.et2050.eu/).

Así pues, se plantea una revisada interrelación y complementariedad entre ámbitos rurales y urbanos (con menores hegemonías de lo urbano y una mayor 'deference' entre ambos), ante una realidad, como la actual, en la que los límites entre ellos resultan cada vez más difusos. Sobre todo en el rural próximo y en el intermedio; pero también en el rural remoto, que basa en las más de las ocasiones su propia existencia en clave de uso, necesidades $\mathrm{y}$ afectos de la población lejana, visitantes y no. Permanece, con todo, la vieja idea de los polos de desarrollo como dinamizadores de sus entornos próximos. Lo que cambian son las condiciones y formas en que esta dinamización se produce, más plural que unívoca de difusión desde el centro. Los territorios, en especial los rurales, pasan a ejercer un papel más proactivo y repolitizado, para convertirse en agentes (pro)activos de su propio desarrollo. Mediante una mayor participación de la población y los Grupos de Acción Local, junto a la administración local, en el diseño, aplicación y seguimiento y evaluación de políticas, planes, programas, proyectos y actuaciones planteados para cada territorio desde los distintos niveles de responsabilidad y competencia.

\section{COMO CONCLUSIÓN}

Hemos visto cómo la gobernanza es un elemento importante en la búsqueda de una mayor cohesión territorial en nuestras sociedades, y también para la creación de las condiciones que permitan una mayor equidad territorial. Así, la cooperación y colaboración

desde el punto de vista europeo o nacional, puede implicar procesos de recentralización o de concentración en las mismas que vayan justamente en contra de un policentrismo real a escala regional y subregional. No tiene por qué ser así, pero si lo fuera, cabe observar que lo importante y más eficiente para la pretendida cohesión y justicia espacial, es justamente el nivel de detalle, las escalas de proximidad. Y es en este sentido que cabe trabajar desde la política y de la planificación.

DRd - Desenvolvimento Regional em debate (ISSNe 2237-9029) 
son elementos de gestión territorial fundamentales para un desarrollo más equilibrado, y como tal quedan recogidos en los documentos oficiales sobre la materia (FARINÓS y GOMIS, 2015). Sin embargo, lo más habitual es que estos acuerdos de colaboración presenten fundamentalmente un carácter meramente interadministrativo, y que su funcionamiento se corresponda con las tradicionales formas de gobierno, no exactamente a fórmulas de gestión territorial y de gobernanza de carácter más relacional, estratégica y proactiva, que resultan más potenciales y que conviene promover con criterio estratégico, democrático y adaptado a cada territorio, pero necesariamente contextualizados dentro del actual marco global.

Un nuevo modelo de desarrollo equilibrado basado en la complementariedad urbanorural deberá contemplar mecanismos de compensación y estructuras flexibles de gobernanza que posibiliten que los municipios del entorno puedan acceder a la actividad económica, los servicios y la innovación gracias al papel que en este sentido pueden jugar las ciudades medias y pequeñas. Romper el predominio de una cultura y tradición más asistencial y clientelar por otra proactiva y propositiva, capaz de promover innovación social e iniciativas autóctonas sobre las que poder sustentar un nuevo desarrollo local alternativo y complementario del actual modelo (que justamente contribuye a explicar este comportamiento), es el reto. Decisión política ('political will') para redoblar esfuerzos en esta dirección resulta crucial, una condición básica y necesaria, aunque no suficiente. Sí si se ve complementada con mayor democracia y empoderamiento para poder decidir el futuro de las comunidades mediante nuevas relaciones, procesos, técnicas e instrumentos de metagobernanza; y una nueva planificación y gestión territorial... de las políticas con impacto territorial... de hecho todas. Unas comunidades ahora con espacios de vida multiterritoriales, intersección entre los diversos niveles escalares en/con los que interactúan. Y es aquí donde la geografía puede aportar su saber hacer para encontrar y adaptar nuevas metodologías y herramientas de análisis. Nueva inteligencia territorial, nuevo desarrollo sostenible, nueva gobernanza para la justicia territorial, a partir de una nueva planificación y gestión del territorio, en especial desde la escala local, de proximidad, con una renovada interpretación del policentrismo y la cooperación urbano-rural con visión sistémica y compleja, son algunos de los elementos principales que han guiado el discurso que el autor ha tratado de ofrecer en este texto.

\section{REFERENCIAS ${ }^{16}$}

ALBRECHTS, L. "Bridging the Gap: From spatial planning to strategic projects". En FARINÓS, J.; ROMERO, J.; SALOM, J. (Eds.) Cohesión e Inteligencia Territorial. Valencia, IIDL/PUV, Colección 'Desarrollo Territorial' n. 7, p. 85-103, 2009.

ALMASA MAZA, B. "Inteligencia territorial para una redefinición eficiente de las políticas públicas”. Trabajo, n. 23, p. 75-94, 2010.

ARRELLANO, D.; SÁNCHEZ, J.; RETANA, B. “¿Uno o varios tipos de gobernanza? Más allá de la gobernanza como moda: la prueba del tránsito organizacional". Cuadernos de Gobierno y Administración Pública I, n. 2, p. 117-137, 2014.

${ }^{16}$ Nota do Editor: Opta-se por manter as referências no estilo utilizado pelo autor. 
BARCA, F. An Agenda for a Reformed Cohesion Policy. A place-based approach to meeting European Union challenges and expectations. Independent Report prepared at the request of Danuta Hübner, Commissioner for Regional Policy, 2009. Disponible en:

$<$ http://ec.europa.eu/regional_policy/archive/policy/future/pdf/report_barca_v0306.pdf > .

BENKO, G. La science régionale. París, Presses Universitaires de France, 1998.

BOZZANO, H. "Geografía e Inteligencia Territorial. Geo-grafein, Geo-explanans, Geotransformare”. Revista Geográfica Digital, IGUNNE, v. 10, n. 19, 2013.

BOZZANO, H. Territorio e Inteligencia Territorial: Geografias, saberes, ciencia y transformación. La Plata, UNLP-CONICET, 2011.

COMISIÓN EUROPEA (CE). La Gobernanza europea. Un libro blanco, COM (2001) 428 final, Bruselas, 2001. Disponible en: $<$ http://eur-lex.europa.eu/legalcontent/ES/TXT/PDF/?uri=CELEX:52001DC0428\&from=ES>.

DAVOUDI, S.; STRANGE, I. "Space and place in the twentieth century planning: An analytical framework and an historical review". En: Davoudi S. and Strange I. (eds.) Conceptions of Space and Place in Strategic Spatial Planning. Londres, Routledge, 2009, p. $7-42$.

FARINÓS, J. (compilador y editor). ESPON PROJECT 2.3.2 Governance of Territorial and Urban Policies from UE to Local Level. Final Report. Luxemburgo, ESPON Project 2.3.2. Publicación electrónica. ESPON Program, 2007. Disponible em: $<$ http://www.espon.eu/export/sites/default/Documents/Projects/ESPON2006Projects/PolicyIm pactProjects/Governance/fr-2.3.2_final_feb2007.pdf>

FARINÓS, J. “Administración y gestión del territorio como potencialidad para el buen gobierno". En Serrano, A. (ed.) Planificación y patrimonio territorial como instrumento para otro desarrollo. Valencia, PUV-Fundicot, 2005a. (en prensa).

FARINÓS, J. “Aménagement et gouvernabilité. Les liens entre rhétorique et pratiques. Une dernière chance pour le projet européen?". L'Information géographique v. 79, n. 1, p. 23-44, $2015 b$.

FARINÓS, J. "Planificación Territorial y Desarrollo Local, y su relación con las nuevas formas de gobernanza territorial asociadas. Un renovado espacio de aplicación profesional", Em: NOGUERA, J. et al. (eds.) Gestión y Promoción del Desarrollo Local. Valencia, IIDL/PUV, 2015c. (en prensa).

FARINÓS, J. "Gobernanza, administración pública y territorio; opciones del localismo. Una mirada desde la Geografía". En: Vicerrectorado de Participación y Proyección Territorial, El desenvolupament territorial valencià. Reflexions entorn de les seues Claus. Valencia, PUV, 2014a. p. 91-102.

FARINÓS, J. "Re-Territorializating Local Development in EU; Local-Based against Globalisation Impacts", En: SALOM, J.; FARINÓS, J. (eds.). Identity and Territorial Character; Reinterpreting Local-Spatial Development. Valencia, IIDL/PUV, 2014b, p. 13-35. (Colección 'Desarrollo Territorial'; 13) 
FARINÓS, J. "Ciudadanos, poder, gobierno y democracia; una forma de relación inestable“. En: SAHUQUILLO, J.L.; MARTÍN CUBAS, J. (eds.) La era de la \#Política2.0. Valencia, Ediciones Casas-AVAPOL, 2014c, p. 24-34.

FARINÓS, J. "Evaluación de políticas y dación de cuentas, requisitos para una adecuada gobernabilidad territorial a partir de una nueva planificación; combinando política y geografía”. Perspectiva Geográfica, v. 20, n. 2, 2014d.

FARINÓS, J. (2013): “Territorial Cooperation as a Means of Achieving Territorial Integration? From Local Place-based to European Union Territorial Cohesion", En: GORZELAK, G.; ZAWALIŃSKA, K. (eds.) European Territories: From Co-operation to Integration. Varsovia, ESPON \& EUROREG - Centre for European Regional and Local Studies, University of Warsaw, 2013. p. 42-53. Diponible en:

$<$ http://www.euroreg.uw.edu.pl/dane/web_euroreg_publications_files/3171/gorzelak_2013_e uropean_territories.pdf.>. Consultado en: 22 ago. 2015

FARINÓS, J. "Inteligencia Territorial para la planificación y la gobernanza democráticas: los observatorios de los territorios". Proyección, n. 5, p. 45-69, 2011 a.

FARINÓS, J. (Ed. y Coord.) (2011b): De la Evaluación Ambiental Estratégica a la Evaluación de Impacto Territorial. Reflexiones acerca de la tarea de evaluación. Valencia, PUV, $2011 \mathrm{~b}$.

FARINÓS, J. “Gobierno, buen gobierno, gobernanza y gobernabilidad de los territorios. Más de lo mismo no será suficiente”, En OLMOS, A et al.. (compiladores) Dinámicas territoriales, políticas de desarrollo territorial sostenible y nueva gobernanza territorial en el espacio Iberoamericano. Conceptos, métodos y tendencias. II Workshop de la RIDOT.

Toluca, México, 10-12 de octubre. 2011c. Publicación en CD: ISBN: 978-607-00-5059-6, 144-175.

FARINÓS, J. “Gobernanza para una renovada planificación territorial estratégica: Hacia la innovación socio-territorial”, Em: MARTÍN, A.; MERINERO, R. (Coords.) Planificación Estratégica Territorial: Estudios Metodológicos. Sevilla, Junta de Andalucía/U. de Jaén/Radeut, 2010. p. 87-113.

FARINÓS, J. "Bases, métodos e instrumentos para el desarrollo y la cohesión territoriales. Diagnóstico y propuestas para el debate y la acción”, En FARINÓS, J.; ROMERO, J.; SALOM, J. (coords.) Cohesión e inteligencia territorial. Dinámicas y procesos para una mejor planificación en la toma de decisiones. Valencia, IIDL/PUV, 2009a, p. 17-62.

(Colección 'Desarrollo Territorial'; 7)

FARINÓS, J. "Cooperación para la cohesión territorial: Una interpretación multinivel desde el SO Europeo", En BOSQUE, J.; RODRÍGUEZ, V.M. (eds.) La perspectiva geográfica ante los retos de la sociedad y el medio ambiente en el contexto ibérico. Alcalá de Henares, Servicio de Publicaciones de la Universidad de Alcalá de Henares, Colección Ponencias del XI Coloquio Ibérico de Geografía, 2009b, p.117-148.

FARINÓS, J. "El reto de una planificación efectiva para poder afrontar los grandes desafíos territoriales en la UE”. En: SÁNCHEZ, L., TROITIÑO, M.A. (coord.), V CIOT. Agua, Territorio y Paisaje. De los instrumentos programados a la planificación aplicada, Madrid: 
Asociación Interprofesional de Ordenación del Territorio (FUNDICOT). Actas del Congreso Internacional de Ordenación del Territorio, 2009c. p. 485-502.

FARINÓS, J. "Gobernanza territorial para el desarrollo sostenible: Estado de la cuestión y agenda". Boletín de la Asociación de Geógrafos Españoles, n. 46, p. 11-32, $2008 \mathrm{a}$.

FARINÓS, J. “Inteligencia para la gobernanza territorial”, En DE SOUZA, Iglesias, A.; SIMANCAS Cruz, (coord.) Sociedad civil organizada y desarrollo sostenible. Santa Cruz de Tenerife, Consejería de Presidencia Justicia y Seguridad, Gobierno de Canarias, 2008b. p. 1933.

FARINÓS, J. ESPON PROJECT 2.3.2 Governance of Territorial and Urban Policies from UE to Local Level. Final Report ESPON Project 2.3.2. Publicación electrónica, 1. 1-372. Luxemburgo, ESPON Program, 2007. Disponible en:

$<$ http://www.espon.eu/export/sites/default/Documents/Projects/ESPON2006Projects/PolicyIm pactProjects/Governance/fr-2.3.2_final_feb2007.pdf $>$

FARINÓS, J. "Nuevas formas de gobernanza para el desarrollo sostenible en el espacio relacional”. Ería, n. 67, p. 219-235, 2005.

FARINÓS, J. "Challenges of Multi-level Governance for Spatial Planning between Local and Regional Levels". Quaderns de Politica Econòmica (Rvta. Electrónica. $2^{\mathrm{a}}$ Época). Special Issue 'European Spatial Planning: A view from Spain', n. 6, p. 81-95, 2004. Valencia, UD Política Económica, Departamento de Economía Aplicada, Universitat de València.

FARINÓS, J. "Reformulación y necesidad de una Nueva Geografía regional Flexible”. Boletín de la AGE, n. 32, p. 53-71, 2001.

FARINÓS, J.; FERRAO, J. “Gobernanza”, Em: LÓPEZ TRIGAL, L. (coord.) Diccionario de Geografia Aplicada y Profesonal. Terminologia de analisis, planificacion y gestion del territorio. León, Universidad de León, 2015.

FARINÓS, J.; GOMIS, A. "La contribución de las fórmulas existentes de cooperación intermunicipal: El caso de la Mancomunitat de La Ribera Alta". Comunicación presentada a las VI Jornadas de Desarrollo Local de la Comunitat Valenciana 'Polítiques de equitat, politiques de desenvolupament: sense equitat no hi ha Desenvolupament', 10 de diciembre, 2015.

FARINÓS, J. ; VERA, O. "Relaciones entre plan y poder (política) ¿la cuadratura del círculo?". Comunicación presentada al IV Workshop de la Red Iberoamericana de Observación Territorial, Eje temático 3: Experiencias de relación entre expertos y políticos en materia de planificación y gestión territorial. Lisboa 30.09-03.10.2015.

GALLEGO, J.R.; PITXER, J.V. "Reinterpretando el desarrollo territorial: una nueva visión desde la economía", En FARINÓS, J. (ed. y coord.) Cómo hacer del territorio cuestión política de Estado / Achieving Territory Becomes Matter of State Importance / Come faire du Territoire une question politique d'État. Valencia, Tirant Lo Blanch, 2015. (en edición).

GIRARDOT, J. "Inteligencia Territorial y Transición Socio-Ecológica". Revista andaluza de relaciones laborales, n. 23, p. 15-39, 2010. 
GRINDLE, M.S. “Good Enough Governance Revisited”. Development Policy Review v. 25, n. 5, p. 553-574, 2007.

HOOGHE, L.; MARKS, G. "Types of multi-level governance". European Integration online Papers (EIoP), v. 5, n. 11, 2001. Edición electrónica: $<$ http://eiop.or.at/eiop/pdf/2001011.pdf $>$

KOOIMAN, J. "Governing as Governance". International Public Management Journal, v. 7, n. 3, p. 439-442, 2003.

LEWIN, K. “Action research and minority problems". Journal of Social Issues, v. 2, n. 4, p. 34-46, 1946.

LINDBERG, L.N.; CAMPBELL, J.L.; HOLLINGSWORTH, J.R. "Economic governance and the analysis of structural change in the American economy", En CAMPBELL, J.L; HOLLINGSWORTH, J.R.; LINDBERG, L.N. (eds.) The Governance of the American Economy. New York, Cambridge University Press, 1991. p. 3-34.

LU, P.; STEAD, D. "Understanding the notion of resilience in spatial planning: A case study of Rotterdam, The Netherlands". Cities, n. 35, p. 200-212, 2013.

MARKS, G.; HOOGHE, L. "Contrasting visions of multi-level governance”, En Bache, I.; Flinders, M. (eds.) Multi-level governance. Oxford, Oxford University Press, 2004, p. 15-30.

MARTÍN CUBAS, J. "La reforma de las administraciones y planificación territorial en España: cómo llevar la política territorial a las instituciones", En: FARINÓS, J. (ed. y coord.) op. cit. ant. Valencia, Tirant Lo Blanch, 2015. (en edición).

MAYNTZ, R. "El Estado y la sociedad civil en la gobernanza moderna". Reforma y Democracia. Revista del CLAD, n. 21, 2001. Disponible en: http://old.clad.org/portal/publicaciones-del-clad/revista-clad-reformademocracia/articulos/021-octubre-2001/0041004

MORATA, F.; HANF, K. Gestión pública y redes de políticas públicas: la gobernación del medio ambiente en Cataluña. Working Paper, Barcelona, Institut de Ciències Polítiques i Socials, 2000.

PASCUAL ESTEVE, J. M. (2011): El papel de la ciudadanía en el auge y decadencia de las ciudades. Valencia, Tirant lo Blanch.

PETERS, B.G. "Governance, Meta-governance”, En: ZURBRIGGEN, C.; NAVARRO, F.M. (coords.) Cambio de política en gestión de redes institucionales, ¿un nuevo modelo de gestión? México, egap/Porrúa, 2010.

PIERRE, J. "Introduction: Understanding governance", En: PIERRE, J. (ed.) Debating governance. Authority, steering, and democracy. Oxford, Oxford University Press, 2000.

PIKE, A.; RODRÍGUEZ-POSE, A.; TOMANEY, J. Desarrollo local y regional. Valencia, Publicaciones de la Universidad de Valencia, 2011. 
PNUD Governance for Sustainable Human Development. Nueva York, UNDP, 1997.

PRATS CATALÀ, J. De la burocracia al management. Del management a la gobernanza. Las transformaciones de las Administraciones públicas de nuestro tiempo. Madrid, INAP, 2005.

PRATS CATALÀ, J. “Gobernabilidad democrática para el desarrollo humano”. Revista Instituciones y Desarrollo, n. 10, p. 103-148, 2001.

RHODES, R.A.W. "Governance and Public Administration”, En: PIERRE, J. (ed.) Debating governance. Authority, steering, and democracy. Oxford, Oxford University Press, 2000.

RHODES, R.A.W. "Understanding Governance”. Buckingham and Philadelphia, Open University Press, 1999.

ROMERO, J.; FARINÓS, J. “Redescubriendo la gobernanza más allá del buen gobierno. Democracia como base, desarrollo territorial como resultado". Boletín de la Asociación de Geógrafos Españoles, n. 56, p. 295-319, 2011.

SÁNCHEZ DE JUAN, J.A. "La coordinación de estrategias territoriales y sectoriales como modelo de inteligencia gubernamental”, Em: ROMERO, J.; FARINÓS, J. (eds.) Ordenación del territorio y desarrollo territorial: el gobierno del territorio en Europa: tradiciones, contextos, culturas y nuevas visiones. Oviedo, Trea, 2004, p. 313-324.

SAPOUNTZAKI, K. "Resilience versus public spatial planning in times of crisis. Lessons from Greece”, En: FARINÓS, J. (ed. y coord.) op. cit. ant. Valencia, Tirant Lo Blanch, 2015. (en edición).

SANTOS, M. La naturaleza del espacio. Técnica y tiempo. Razón y emoción. Barcelona: Ariel, 1996.

STOKER, G. Governance as theory: five propositions. International Social Science Journal, v. 50, n. 155 , p. $17-28,1998$.

SUBIRATS, J. "La política entre los argumentos de la razón y las exigencias de la democracia. Acerca de las decisiones públicas", En: FARINÓS, J. (ed. y coord.) op. cit. ant. Valencia, Tirant Lo Blanch, 2009. (en edición).

TUTISTAR, D.; PINAZO-DALLENBACH, P. "Prioridades de ajuste y mejoramiento de la administración pública en Colombia: Análisis crítico a dos estrategias de gestión".

Comunicación presentada al IV Workshoop de la Red Iberoamericana de Observación Territorial -RIDOT- 'El reto de la planificación y observación territorial en Iberoamérica para el siglo XXI: dinámicas, procesos, experiencias y propuestas', Lisboa, 1-3 de octubre, 2015.

VALENZUELA-MONTES, L.M.; CARVALHO-CORTES, J. "Observatorios urbanos en América Latina: ¿observar o participar?”. Economía, Sociedad y Territorio v. 15, n. 49, p. 779-806, 2015. 
VALENZUELA-MONTES, L.M.; SORIA, J.A. “Observatorios territoriales y urbanos en Europa ¿entidades pasivas o instrumentos operativos para la planificación?”. Ciudad y territorio: estudios territoriales, n. 168, p. 243-260, 2011.

WEISS, T.G.. "Governance, Good Governance and Global Governance: Conceptual and Actual Challenges". Third World Quarterly, v. 21, n. 5, p. 795-814, 2000.

WORD BANK. Economic Growth in the 1990s: Learning from a Decade of Reform. Washington, D.C., The International Bank for Reconstruction and Development / The World Bank, 2005. Disponible en:

$<$ http://www1.worldbank.org/prem/lessons1990s/chaps/frontmatter.pdf>

ZURBRIGGEN, C. “Gobernanza: una mirada desde América Latina”. Perfiles latinoamericanos, v. 19, n. 38, p. 39-64, 2011.

Artigo recebido em: 21/09/2015

Artigo aprovado em: 08/10/2015 